\title{
Research on the mechanism of Low pressure economizer wet fouling shape
}

\author{
Bingwen Zhang ${ }^{a}$, Zhigang Zhang ${ }^{\text {b }}$ \\ School of Northeast Dianli University, Jilin 132012, China \\ a340002197@qq.com, bzg1988.happy@163.com
}

Keywords: low pressure economizer; cleaning device; dew point; wet fouling.

\begin{abstract}
Since there has been no feasible way to remove wet flue gas of coal heating surface fouling, modern coal technology forced to raise the temperature of flue gas emissions, keep the flue gas temperature above the dew point of the flue gas acid run, so the flue gas heat abandoned lead a lot of waste. Installation in low pressure economizer cleaning device that can effectively solve the problem of fouling and plugging ash coal flue gas conditions under condensation heating surface, breaking the flue gas acid dew point restrictions on heat utilization. Analysis wet fouling shape generation and migration, and the establishment of wet fouling shape model derived shape description, and then find out the relationship between the thickness of wet fouling and heat transfer coefficient for the study of lower exhaust gas temperature, further use of waste heat is of great significance .
\end{abstract}

\section{Introduction}

Among coal-fired boiler heat loss in smoke heat loss accounts for the biggest, about $60 \% \sim 70 \%$ of the heat loss. Of the important factors influencing the exhaust heat loss exhaust problem is too high, as is often the case, every add the exhaust temperature of $10{ }^{\circ} \mathrm{C}$, exhaust heat loss increased by $0.6 \%$ $\sim 1.0 \%$, and the corresponding coal consumption by $1.2 \% \sim 2.4 \%^{[1-3]}$. Therefore reduce exhaust smoke temperature is good for power plant fuel economy and environmental protection. But because of the particularity of their work environment, present the low-pressure economizer heating surface acid corrosion at low temperature and wet dust phenomenon, using acid materials can effectively solve the problem of sulfuric acid corrosion of heating surface ${ }^{[4]}$, but because the removal of wet dust collecting and cleaning dry ash is a very different nature, wet dust removal is still not solved ${ }^{[5-6]}$.So the increase in the current low pressure economizer system corresponding to the wet dust ash removal device, breakthrough the limitation of coal-fired flue gas acid dew point of thermal energy utilization, to further improve the efficiency of the boiler ${ }^{[7-10]}$.

\section{Wet dust formation and the trend}

In wet dust surface below the premise of the flue gas acid dew point temperature, condensation environment can be divided into the flue gas temperature is higher than sulfuric acid dew point and the flue gas temperature is lower than the sulfuric acid dew point two cases for analysis.

Under the condition of the flue gas temperature is higher than the sulfuric acid dew point, the sulfuric acid as gas in flue gas, only when the sulfuric acid gas molecules into the boundary layer (laminar bottom) close to even touch the wet dust on the surface of the time, only has the condition of condensation.

Under the condition of the flue gas temperature is lower than the sulfuric acid dew point, the flue gas of sulfuric acid as liquid state or fog, is actually a range of particle size of droplet. Total sulfur acid bead in the flue gas can use arb sulfur content of coal and water estimates. In a specific environment, the number of sulfur acid bead will adhesion to the surface of a wet dust, is one of the main characteristics of wet dust description[11-13]. In a typical staggered tube bundle of heating surface, the flue in the tube bundle is bend rules, trace of flue gas molecules is continuous, and not with wet dust collecting surface intersection. For sulfur acid bead size is small enough, due to the large surface area, volume is relatively small, relatively less mass, flue gas friction is rising as the leading force, the inertia force of sulfur acid bead friction and gravity drop for flue gas of 
infinitesimal, sulfur acid bead fettered by friction, along with the smoke movement. So, in this condition, sulfur acid bead trace trace overlap with flue gas molecules, sulfur acid bead not clung to the wet dust on the surface. For sulfur acid bead size bigger, because of the sulfur acid bead order of magnitude with flue gas friction, inertia force and gravity or rise to dominance, sulfur acid beads will be free of flue gas friction's at the corner of the flue gas, may impact the wet dust captured on the surface, especially in wet flue gas dust do near the surface of the large turning Angle, for example, after the orthogonal windward side of the heating pipe, heating pipe vortex area.

Flue gas of fly ash particles adhesion on wet dust collecting surface similar to the process of sulfur acid bead, but because of different density, different surface shape and is slightly different[11-13].In fact, when the flue gas temperature is lower than under the condition of the sulfuric acid dew point, parts of the flue gas sulfur acid beads are often wrapped in fly ash particles, that is to say, the fly ash particles with sulfuric acid bead, appearance is a ball. Gravity, viscous force, condensing core can be explained by the fly ash particles and the intrinsic reason sulfur acid bead together.

\section{The mechanism modeling design of wet dust on heating surface tube}

Wet dust shape under the influence of the ash removal device movement (Regardless of the gravity and flue gas friction). The runtime set soot cleaning device. Start, before and after the slag discharge device as stained with thickness of $\Delta \mathrm{h}(\mathrm{mm})$ of wet dust shape, as shown in figure 1.

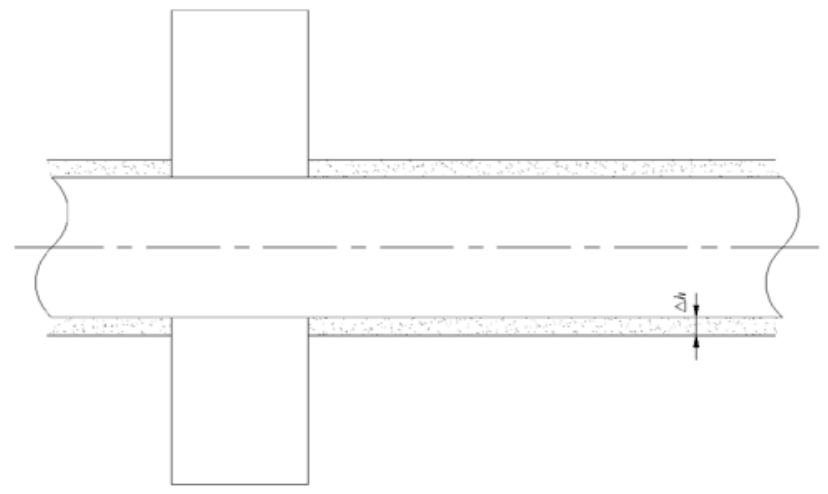

Figure 1 the appearance of wet dust

Without considering the gravity and friction under the condition of flue gas, soot cleaning device as start $\Delta \mathrm{t}(\mathrm{s})$ after the wet dust shape for a quarter of a circle, as shown in figure 2. This is the largest heavy appearance, can use the equation of circle (type (4)) describe the boundary coordinates. Diagram, two point line for ash removal device before the start position.Wet dust within the micro square said ash yuan. Based on two principles:

(1) The shortest distance of each gray infinitesimal;

(2) Volume constant (ash and liquid are considered to be incompressible body), that is, the total volume constant before and after the migration, each grey infinitesimal volume before and after the migration.

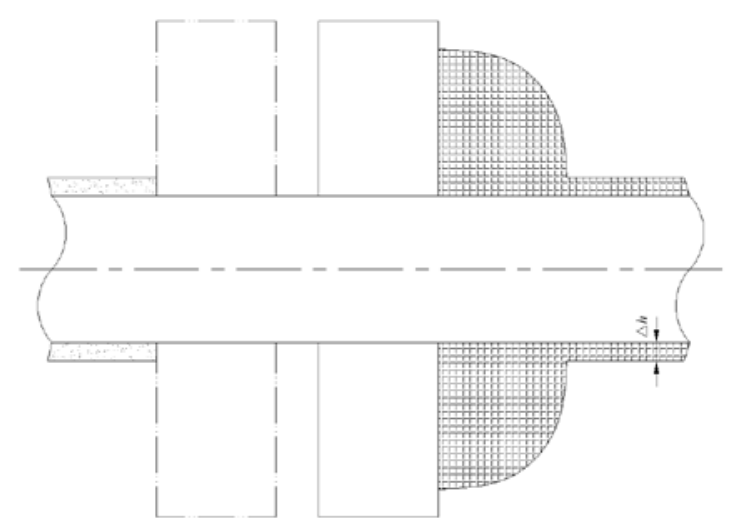

Figure 2 the appearance of wet dust 
Wet dust shape under the influence of gravity. Establish a cylindrical coordinate system after the migration of the triangle area, as shown in figure 3. Right Angle of the triangle area position $\mathrm{z}=0$ points for cylindrical coordinate system. The gravity of every grey micro yuan in grey tube adhesive surface shear force and bending moment. Shear bonding surface displacement trend, grey tube bending moment make the grey tube adhesive surface has open trend. Grey micro yuan for ash tube on the surface of the adhesive shear force and bending moment for each of the ash to small yuan because of gravity on the grey tube adhesive surface shear force and bending moment together. From micro yuan, ash pipe on the surface of the adhesive ashes began to calculate shear force and bending moment and accumulation. Set the first k grey micro yuan, grey infinitesimal combined shear force is greater than the friction (N) or gravity is greater than the viscous ability, is the first (k-1), a wet dust ash micro yuan of the outer gray infinitesimal, the grey infinitesimal geometry center sat marked $(\theta \mathrm{s}$, rs, zs), its collection is under the influence of gravity, wet dust three-dimensional shape description. Among friction power $(\mathrm{N})$ for the grey and grey micro yuan with maximum static friction force between ash tube adhesive surface, its direction and the vertical diameter. Viscous ability among (N) for the grey and grey micro yuan and grey tube maximum bonding strength between adhesive surface, the direction parallel to the pole diameter, and reverse strengthening, synthetic diminished.

Assume the following:

(1) The grey micro yuan for incompressible body

(2) Didn't consider different $\mathrm{z}$ and coordinate position among the ashes of $\theta$ reaction.

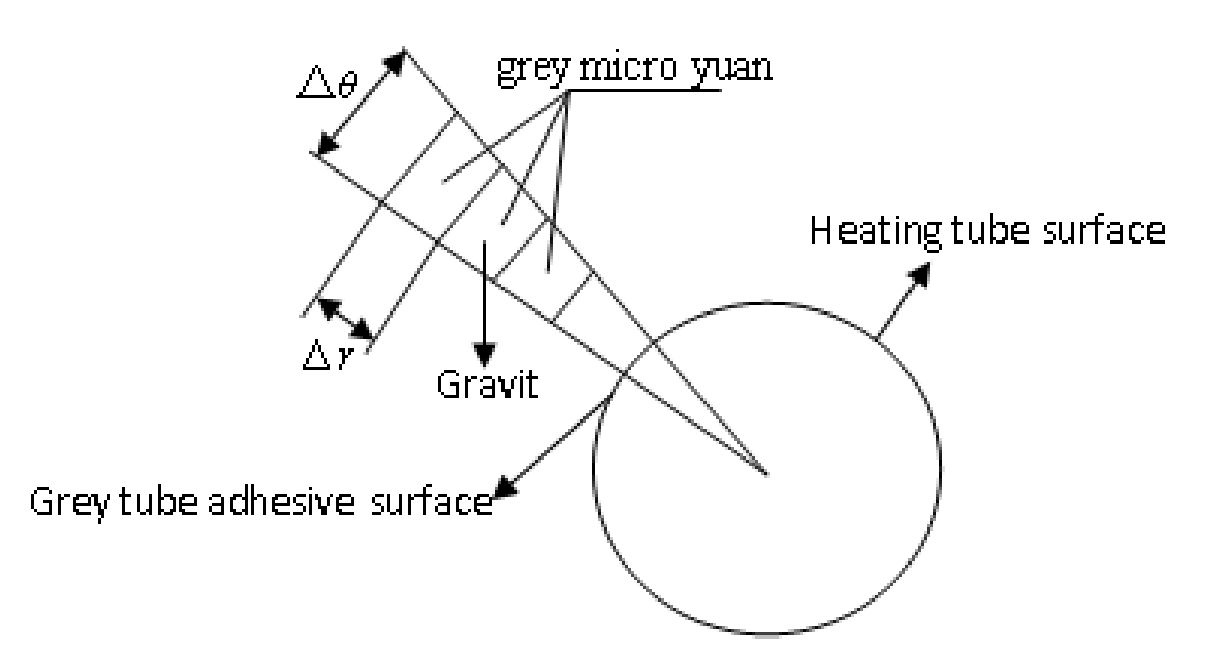

Figure 3 cylindrical coordinate system

Gravity and flue gas wet dust shape under the influence of the friction force. On the basis of the gravity and to the wet dust the surface ash infinitesimal friction, coupled with flue gas will be produced under the double influence of gravity and the friction flue gas wet dust three-dimensional shape description. Calculation and gravity simultaneously, but not to the cumulative gas friction force.

The main expression. Considering the dynamic process. Deposited ash removal device start after 1 second, the origin of coordinates to form a quarter ring, circle center in the center of the pipe line, circle tube center on the origin of coordinates; Ash removal device start after 2 seconds, added after the migration of grey dust in a quarter of the origin of coordinates to form a larger ring;.......When migration filling balance of formation of fixed shape. Ash removal device to start the first seconds after i the heavy volume of Qi (mm3) with the first i-1 seconds after the heavy volume of Qi-1 by type (1) the relation between 1 said.

$Q_{i}=Q_{i-1}+\pi V \Delta h\left(D_{\mathrm{w}}+\Delta h\right)-\Delta Q$

As $\Delta Q$ - migration volume $\left(\mathrm{mm}^{3}\right)$

1 seconds after the heavy volume of Qi (mm3) by type (2)

$Q_{1}=\pi V \Delta h\left(D_{\mathrm{w}}+\Delta h\right)$ 
The accumulation of the i seconds from $\mathrm{Li}$ (from the origin along the pipe axis direction) by equal volume and (in the absence of gravity and friction, the accumulation volume for a quarter of a circle), by type (3)

$$
\frac{\pi^{2} L_{i}^{2}\left(D_{\mathrm{w}}+L_{i}\right)}{4}=Q_{i}
$$

The equation of circle on biggest border by type (4)

$$
z^{2}+\left(y-\Delta h-D_{\mathrm{w}} / 2\right)^{2}=L_{i}^{2}
$$

Ash removal device surface of ash yuan also prevent grey cell migration, adhesion of the adhesive force in the form of the shear stress transfer to calculate grey micro yuan. Among from the ash removal device to calculate ash ability of any surface shear force than the gray cell migration.

The driving force for the ash removal device. On the tube of heating surface appearance, grey cell adhesion to the surface of the tube in the sum of the heating surface tube axis direction projection is the driving force of soot cleaning device is needed. In fact, this force is grey and rough on the surface of the metal surface friction, experience the friction coefficient is $0.7 \sim 0.9$.Gray surface and roughness on the surface of the metal friction is greater than the gray surface and the shear stress on the surface of the ash, and the difference is bigger, especially considering the soot formation has the nature of the fluid, the lack of resistance to shear force.

\section{Modeling design analysis}

Using C language programming software to build models, $\mathrm{Dh}(\mathrm{mm})$ are deposited in the ash removal device before the start of known thickness, the ash removal device speed $\mathrm{V}(\mathrm{mm} / \mathrm{s})$, are deposited by seconds after launch wet appearance mechanism of the numerical solution of the model.(obtained: pipe diameter Dw(mm), the thickness of the deposited soot cleaning device before the start of Dh $(\mathrm{mm})$, the ash removal device speed $\mathrm{V}(\mathrm{mm} / \mathrm{s})$, flue gas velocity $\mathrm{Vg}(\mathrm{mm} / \mathrm{s})$, the density of grey sh $(\mathrm{kg} / \mathrm{m} 3)$, flue gas dynamic viscosity uy (Pa s.), heavy dynamic viscosity ua $(\mathrm{Pa} \cdot \mathrm{s})$, flue gas boundary layer thickness $\mathrm{h}(\mathrm{mm})$, three coordinates, the direction of the step; finally the output current grey infinitesimal coordinates (st, $r, z$ ) to a file).

Reuse CAD drawing software of soot cleaning operation in pattern. figure4, figure 5 of ash removal device respectively after start 1 second and 2 seconds.
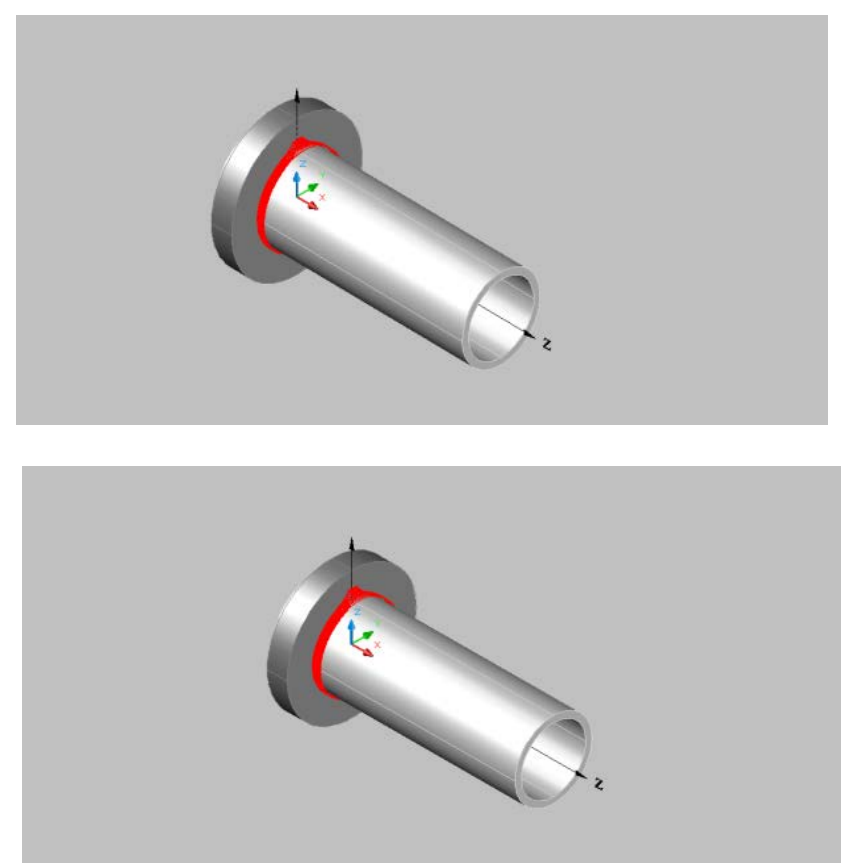

Figure 4(5) ash removal device start graphics after 1 second(2 seconds) 


\section{Conclusion}

(1) on the low pressure economizer tube with soot cleaning device (ash removal device) can effectively remove dust damp, reveals the clear the objective laws of wet dust, can effectively solve the soot under the condition of condensation gas heating surface dust and ash problem.

(2) to improve the design principle of the thermal energy utilization system "to avoid condensation" to "allow condensation", breakthrough the limitation of coal-fired flue gas acid dew point of thermal energy utilization.

(3) in combination with the practical situation of the scene, tailor special driving devices for soot cleaning device, it can be applied to thermal power plants, industrial boiler and heating system, energy conservation and emissions reduction effect and broad prospect of application.

\section{References}

[1] Yan Shunlin,Li Yonghua,et al.Classification and Analyses of the Causes of Exit Gas Temperature Increasing Utility Boiler [J].Electric Power,2000/6,33(6):20-22(in Chinese).

[2] Xiao Lichuang,Li Ming, Jinling petrochemical thermal power plant \# 3 the analysis and countermeasures of coal-fired boiler exhaust smoke temperature is on the high side [J].Journal of Jiangsu Institute of Petrochmical Technology,2000/9,12(3):46-49(in Chinese).

[3] Zeng Xiaozhong.Analysis and Countem easure on Boiler Exhaust Gas Superheating in Cogeneration Power Plant[J].North China Electric Poewr,2004,3 (3):44-46(in Chinese).

[4] Wang Yan.Application of the Fluoroplastic Low Temperature Economizer in the Coal-fired Power Plant[J].Energy and Energy Conservation,2013,92(5):119-120(in Chinese).

[5] Xin Quzhen,Kang Da,Jiang Sen.Analysis of Key Technology in Boiler Flue Gas Waste Heat Recovery System[J].Power System Engineering,2011,27(3):58-58(in Chinese).

[6] XU Gang, XU Cheng, YANG Yongping,et al.Deep Utilization of Exhaust Heat and Comprehensive Optimization of Tail Heating Surfaces for Utility Boilers[J] .Proceedings of the CSEE,2013,33(14):1-8 (in Chinese).

[7] Zhang Bingwen, Yang Ping,Zhou Zhenqi,et al.A new type of low temperature economizer in the application of power plant boiler[J].Energy Conservation,2002,242(9):32-33(in Chinese).

[8] Zhang Bingwen, Hu Sike.Utilization of Heat Energy in Flue Gas to Save Energy and Reduce Production Cost[J].Power System Engineering,2004,20(1):28-29(in Chinese).

[9] Zhang Bingwen,Zhou Zhenqi.Method to Utilize the Heat in the Flue Gas from Boilers of Coal-fired Power Plants[J].Boiler Technology,2010,41(2):58-60(in Chinese).

[10] Zhang Bingwen,Zhai Xiurong,Wang Xuelian. Method to Reduce Coal\}ired Boiler Smoke Temperature[J].Chemical Engineering \& Machinery,2013,40(3):316-318(in Chinese).

[11] Yuanyuan Shao, Jinsheng Wang, Chunbao (Charles) Xu,et al. An experimental and modeling study of ash deposition behaviour for co-firing peat with lignite[J].Applied Energy, 2011, 88:2635-2640.

[12] Chao Luan, Changfu You, Dongke Zhang. An experimental investigation into the characteristics and deposition mechanism of high-viscosity coal ash[J].Fuel,2014,119:14-20.

[13] Yadi Pan, Fengqi Si, Zhigao Xu,et al. An integrated theoretical fouling model for convective heating surfaces in coal-fired boilers[J].Powder Technology,2011,210:150-156. 